\title{
On the Application of Internet of Things (IOT) in Cold Chain Logistics Management
}

\author{
Qingqiang Huang ${ }^{a}$, Xuehu Yan \\ Guilin University of Electronic Technology, Beihai Campus, P. R. China, 536000, China. \\ a45306174@qq.com
}

\begin{abstract}
Characterized by big data, artificial intelligence, IOT, automation, the industrial sector's transition to Industry 4.0 is much more than a question of technology. It will affect not only individual corporate strategy but also national economic and employment policy. Cold chain logistics will also witness great changes with IOT, with which a full Logistic traceability system can be established. Factors of quality and safety of agricultural products and perishables run throughout their whole logistic processes from production to processing, packaging, storage, transportation and sales. Based on the analysis of the connotation of the cold chain logistics system, this article puts forward a mode of cold chain logistics traceable system's framework and key elements, and further analyzes its trends and merits it brings about on cold chain logistics.
\end{abstract}

Keywords: Application, IOT, Cold-chain Logistics, Benefits.

\section{Introduction to IOT and Cold Chain Logistics}

\subsection{Internet of Things (IOT)}

IOT is short for internet of things. In a sense, The Internet of Things refers to the

ever-growing network of physical objects in our life that all feature its own IP address for internet connectivity, and the communication that occurs between these objects and other Internet-enabled devices and systems.

\subsection{Cold Chain Logistics}

Cold chain logistics includes all of the means used to ensure a constant temperature for a product that is not heat stable, from the time it is manufactured until the time it is used. Moreover, cold chain is considered as a science, a technology and a process. It is a science as it requires the understanding of the chemical and biological processes associated with product perishability. It is a technology as it relies on physical means to ensure desirable temperature conditions along the supply chain. It is a process as a series of tasks must be performed to manufacture, store, transport and monitor temperature sensitive products.

\subsection{IOT Platform in Cold Chain Logistics: Intelligent, Compatible, Versatile}

An IOT resolution offers a powerful data-link platform with multi-sensor smart

tracking devices, and highly innovative software applications, enabling us to monitor all cargo and containers across the globe in real time for maximum security, guaranteed quality and total visibility. ( Typical case seen in Figure One ) 


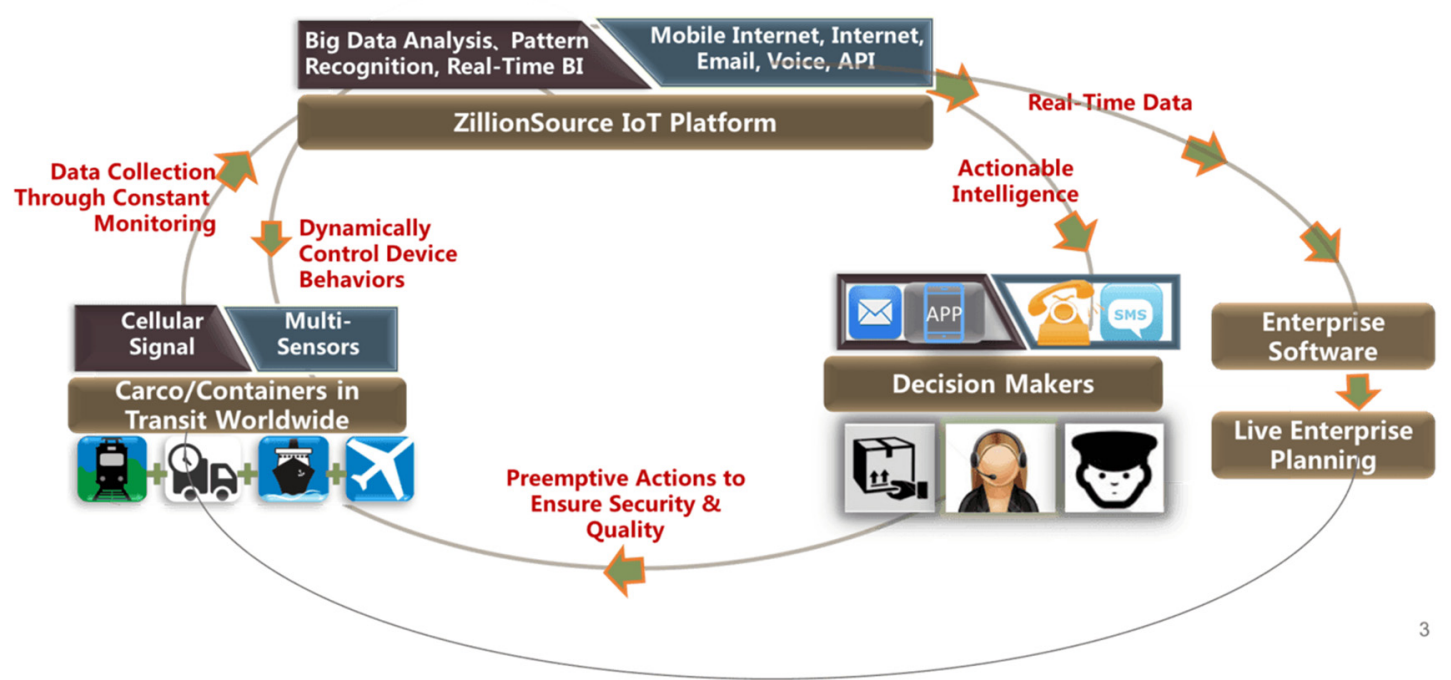

Figure 1. An IOT Platform offered by ZillionSource Technologies Co. Ltd.

A typical IOT platform should be intelligent, compatible and versatile. As for versatile, the platform should support large spectrum of data types,; and function in many aspects or sectors in the society, such as logistics, health care, petroleum, natural gas, smart home, etc; and be applicable to rules setting for different types of data and scenarios to offer customrized needs for different commercial and business clients. With regards to intelligent, the platform should have stellar pattern recognition capabilities for analyzing streaming data to provide real-time insights, helping clients make better business decisions. For compatible, the platform should be compatible with many different hardware devices, users and platforms and handle massive amount of data.

\section{IOT and Traceability System}

The design and implementation of agricultural products traceability system based on IOT improve the credibility in the circulation process of meat and vegetables, standardize enterprise production management, and enable consumers to obtain the right to know. Web technology, bar code technology, data collecting middleware technology is used for the construction of database server platform and SOA-oriented software information system. Traceability code and data standard protocol of noncontact IC cards are also developed to improve the urban network topology and establish a complete coverage of all aspects of the circulation, such as electronic transaction settlement, quality testing and supervision, real-time data collection and products sources tracing. Thus, an end-to-end traceability system can link the flow of information to physical products. In an event of a food outbreak, the sharing of traceability information between trading partners in the supply chain is critical to ensure that a targeted and effective recall is conducted. Also standards offer globally unique identification and data capture of trade items, assets, logistic units, parties and locations. In addition, all standards enable critical data exchanges to be recorded so that there is alignment of traceability information between trading partners. (A typical cold chain logistics traceable system can be seen in Figure 2) 


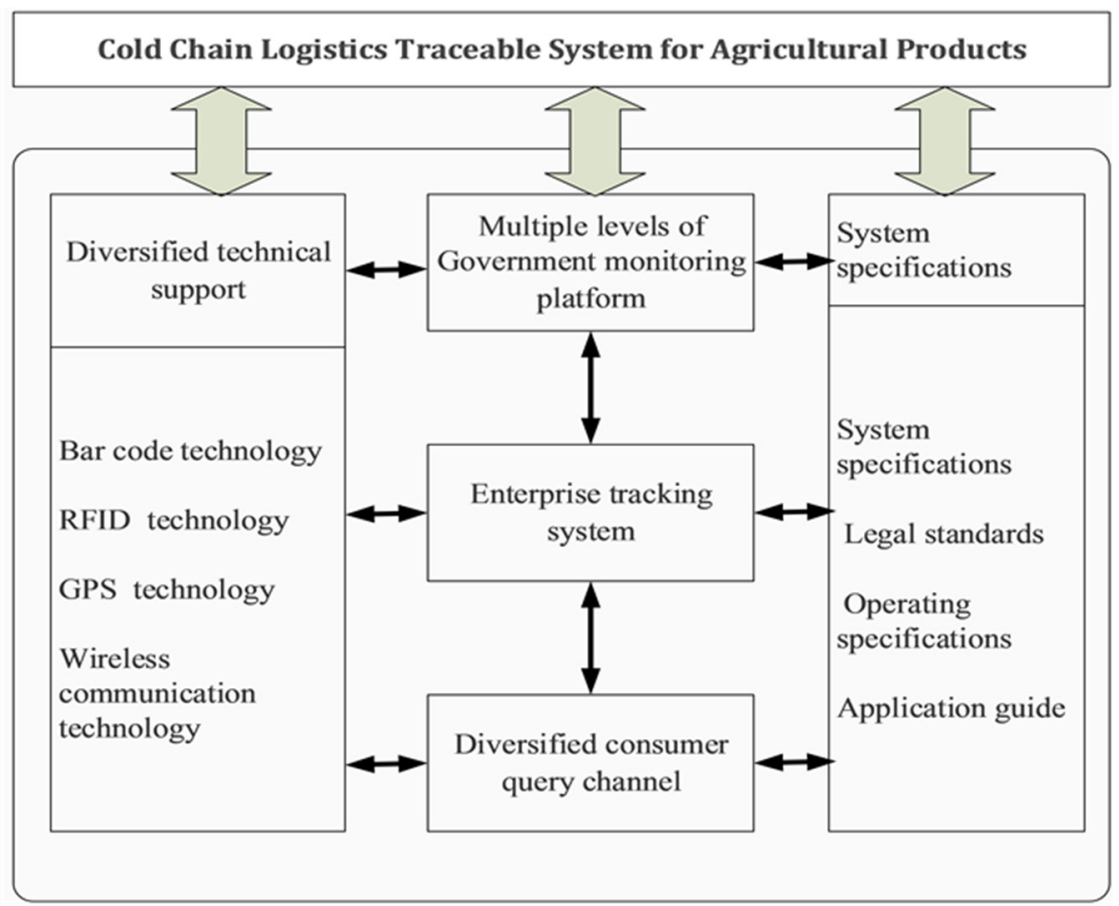

Figure 2. typical cold chain logistics traceable system

\section{Benefits Bought by IOT on Cold Chain Logistics}

\subsection{IOT Minimizes Error and Risk, Reduce Loss}

Traditionally, supply chain transactions are completed manually, creating delays and a higher risk for recording error, which can cause differences between what was recorded and what was actually loaded. By digitizing this process using IOT, the relevant information is captured directly from the sensors placed around the products, and entered onto the platform, creating a single, shared repository that all authorized participants can access and which can only be altered with consensus from all parties.

\subsection{IOT Protects Assets and Cargoes}

The tracking solution is designed to monitor what is happening with the items, captures the input and output weight to define available capacity, in addition to identifying which silo and person will carry the load. The data is then correlated against external information, such as weather, humidity, temperature and the driver's data, providing customers with a much more accurate delivery time estimate.

\subsection{IOT Ensures Item Quality of Perishables}

Knowing and recording the temperature of the perishables during every step of the cold chain holds every party involved in the process accountable. It provides end users an unbroken chain of measurements and associated uncertainties, enabling designers to trace the accuracy level of their end product's temperature measurements

In this way, all perishables are of good quality throughout the whole end-to-end process.

\section{Outlook of IOT in Cold Chain Logistics}

Advances in IOT will enable unprecedented optimisation of cold chain logistic business processes: from tracking goods to remote management of equipment and fleets, predictive maintenance and more. Companies will benefit from enhanced security for their teams and traceability for their assets, as well as improved productivity and competitiveness - and therefore better control over quality and costs. 
Employees will benefit from greater security and efficiency while out in the field and predictive support for a number of tasks. With connected and smarter workspaces, they'll have more time to devote to the priorities where they can be most valuable.

IOT will reinvent the relationship between companies and customers: enabling ever more accurate understanding of customer needs, more adapted offers and the appearance of more useful and relevant products and services.

For food companies, risk-based preventive approach that effectively addresses operational, regulatory, and reputational risks is critical for protecting brand. An integrated solution that provides real-time temperature monitoring and product location traceability can help businesses comply with multiple compliance standards and requirements, in addition to internal quality processes. At a time when the food industry as a whole is under significant pressure to regain customer trust, build brand loyalty and protect brand integrity, businesses need to think about innovative solutions. With a preventive program in place, organizations can ensure that consistently high-quality products reach their customers while protecting against cold chain failures and costly recalls, and minimizing the time spent on food safety issues.

In short, IOT facilitates cold chain logistics. And cold chain logistics will get a brilliant future with IOT.

\section{References}

[1]. Wu, D., Lu, C.Y. and Cheng, H. (2009) Food Quality and Safety Traceable System's Research Progress and Application Prospect of Tea Industry. Agricultural Bulletin, 25, 251-255. [Citation Time(s):1].

[2]. Zheng, H.G. (2012) Food Safety Traceable Systems Research. Chinese Academy of Agricultural Sciences, Beijing. [Citation Time(s):1].

[3]. Zhao, Y.J. (2012) Development Situation of China's Food Quality and Safety Traceable System. Jilin Agriculture, 2, 200. [Citation Time(s):2].

[4]. Ma, Y. (2011) Honey Quality Traceable System Based on AGENT. Chinese Academy of Agricultural Sciences, Beijing. [Citation Time(s):1].

[5]. Pelletier W, Brecht JK, do Nascimento Nunes MC, Emond J-P. 2011. Quality of strawberries shipped by truck from California to Florida as influenced by postharvest temperature management practices. HortTechnology21, 482-493. 